\title{
An unexplained exacerbation of depression, anxiety, and panic
}

Rebecca Swenson, PMHNP-BC, FNP-BC

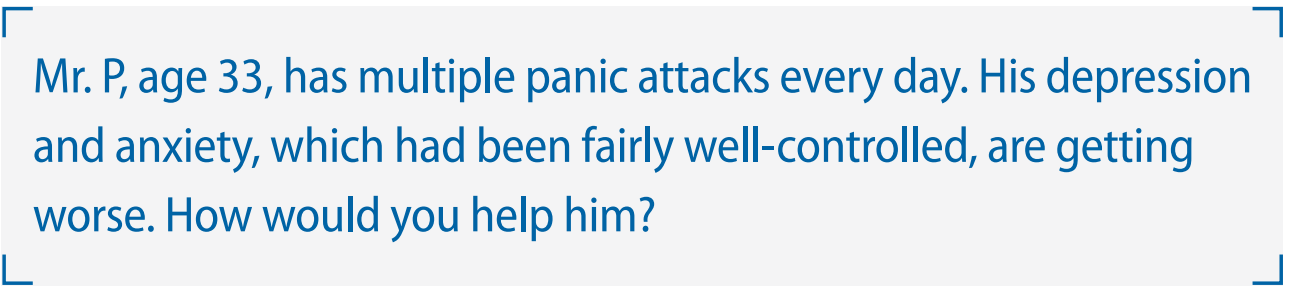

\section{CASE Depression, anxiety, and panic attacks}

At the urging of his parents Mr. P, age 33, presents to the partial hospitalization program (PHP) for worsening depression and anxiety, daily panic attacks with accompanying diaphoresis and headache, and the possibility that he may have taken an overdose of zolpidem. Mr. P denies taking an intentional overdose of zolpidem, claiming instead that he was having a sleep-walking episode and did not realize how many pills he took.

In addition to daily panic attacks, Mr. P reports having trouble falling asleep, overwhelming sadness, and daily passive suicidal ideation without a plan or active intent.

Mr. $P$ cannot identify a specific trigger to this most recent exacerbation of depressed/anxious mood, but instead describes it as slowly building over the past 6 to 8 months. Mr. P says the panic attacks occur without warning and states, "I feel like my heart is going to jump out of my chest; I get a terrible headache, and I sweat like crazy. Sometimes I just feel like I'm about to pass out or die." Although these episodes had been present for approximately 2 years, they now occur almost daily.

\section{HISTORY Inconsistent adherence} For the last year, Mr. P had been taking alprazolam, $0.5 \mathrm{mg}$ twice daily, and paroxetine,
$20 \mathrm{mg} / \mathrm{d}$, and these medications provided moderate relief of his depressive/anxious symptoms. However, he stopped taking both medications approximately 3 or 4 weeks ago when he ran out. He also takes propranolol, $20 \mathrm{mg} / \mathrm{d}$, sporadically, for hypertension. In the past, he had been prescribed carvedilol, clonidine, and lisinopril-all with varying degrees of relief of his hypertension. He denies a family history of hypertension or any other chronic or acute health problems. He reports that he has been sober from alcohol for 19 months but smokes 1 to 2 marijuana cigarettes a day.

\section{EVALUATION Elevated blood pressure and pulse}

Mr. P's physical examination and medical review of systems are unremarkable, exceptfor an elevated blood pressure $(190 / 110 \mathrm{~mm} \mathrm{Hg})$ and pulse ( 92 beats per minute); he also has a headache. A repeat blood pressure test later in the day is $172 / 94 \mathrm{~mm} \mathrm{Hg}$, with a pulse of 100 beats per minute. His urine drug screen is positive only for delta-9tetrahydrocannabinol (THC).

continued

Ms. Swenson is an Advanced Practice Nurse Practitioner, Broadstep Behavioral Health, Milwaukee, Wisconsin.

Disclosure

The author reports no financial relationships with any companies whose products are mentioned in this article, or with manufacturers of competing products.

\section{How would you} handle this case?

Answer the challenge questions at MDedge.com/ psychiatry and see how your colleagues responded 


\section{Clinical Point}

\section{Epinephrine and norepinephrine are produced in excess by an adrenal or extra- adrenal tumor seen in pheochromocytoma}

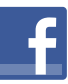

Discuss this article at www.facebook.com/ MDedgePsychiatry

\section{Table 1}

\section{Symptoms of pheochromocytoma}

\begin{tabular}{|l|l|}
\hline More common & Less common \\
\hline Hypertension & $\begin{array}{l}\text { Abdominal pain, } \\
\text { nausea, vomiting, } \\
\text { and diarrhea }\end{array}$ \\
\hline Sweating & Pallor \\
\hline Headache & Hallucinations \\
\hline $\begin{array}{l}\text { Anxiety and panic } \\
\text { (approximately 30\% } \\
\text { of patients) }\end{array}$ & Agitation/apprehension \\
\hline & \\
\hline Sources: References 5-7 & \\
\hline
\end{tabular}

\section{Table 2}

\section{Pheochromocytoma:}

\section{A rare, often-missed diagnosis}

Annual incidence in the general population is 2 to 8 cases per 1 million people $(.000002 \%$ to $.000008 \%)$

In patients with hypertension, the annual incidence is $0.1 \%$ to $1.0 \%$

In patients with adrenal mass, the annual incidence is $5 \%$

Age: 30 s to 50 s

Men and women are equally affected

Autopsy studies suggest that $50 \%$ of pheochromocytomas go undiagnosed

Sources: References 6,8

\section{Table 3}

\section{Causes of false-positive results on serum metanephrines testing}

\begin{tabular}{|l|l} 
Prescription medications & Other \\
\hline Tricyclic antidepressants & Acetaminophen \\
\hline $\begin{array}{l}\text { Monoamine oxidase } \\
\text { inhibitors }\end{array}$ & Caffeine \\
\hline Phenothiazines & Nicotine \\
\hline Buspirone & Alcohol withdrawal \\
\hline Beta-blockers & $\begin{array}{l}\text { Clonidine } \\
\text { withdrawal }\end{array}$ \\
\hline Source: References 6,7 & \\
\hline
\end{tabular}

Which tests should be ordered to rule out a serious medical condition mimicking depression, anxiety, and/or panic attacks?

a) complete blood count (CBC) with

differential

b) thyroid-stimulating hormone (TSH) level with reflex

c) porphyrin tests

d) serum metanephrines test

\section{The author's observations}

A CBC with differential is helpful for ruling out infection and anemia as causes of anxiety and depression. ${ }^{1}$ In Mr. P's case, there were no concerning symptoms that pointed to anemia or infection as likely causes of his anxiety, depression, or panic attacks. A TSH level also would be reasonable, because hyperthyroidism can present as anxiety, while hyperthyroidism or hypothyroidism each can present as depression. ${ }^{1}$ However, both Mr. P's medical history and physical examination were not concerning for thyroid disease, making it unlikely that he had either of those conditions. A review of Mr. P's medical records indicated that within the past 6 months, his primary care physician (PCP) had ordered a CBC and TSH test; the results of both were within normal limits.

Serum porphyrin tests can exclude porphyria as a contributor to Mr. P's anxiety and depression. Porphyrias are a group of 8 inherited disorders that involve accumulation of heme precursors (porphyrins) in the CNS and subcutaneous tissue. ${ }^{2}$ Collectively, porphyrias affect approximately 1 in 200,000 people. ${ }^{2}$ Anxiety and depression are strongly associated with porphyria, but do not occur secondary to the illness; depression and anxiety appear to be intrinsic personality features in people with porphyria. ${ }^{3}$ Skin lesions and abdominal pain are the most common symptoms, ${ }^{3}$ and there is a higher incidence of hypertension in people with porphyria than in the general population. ${ }^{4} \mathrm{Mr}$. P does 


\section{Mr. P's serum metanephrines test results}

\begin{tabular}{|c|c|c|}
\hline Metabolite & Mr. P's results & Interpretation \\
\hline \multirow[t]{2}{*}{ Normetanephrine } & \multirow[t]{2}{*}{$\begin{array}{l}0.93 \mathrm{nmol} / \mathrm{L} \text { (reference range: } \\
0.00 \text { to } 0.89 \text { ) }\end{array}$} & $\begin{array}{l}\text { A value of }>2.2 \mathrm{nmol} / \mathrm{L} \text { is confirmatory for } \\
\text { pheochromocytoma }\end{array}$ \\
\hline & & A value of $<0.9$ excludes pheochromocytoma ${ }^{9}$ \\
\hline \multirow[t]{2}{*}{ Metanephrines } & \multirow{2}{*}{$\begin{array}{l}0.54 \mathrm{nmol} / \mathrm{L} \text { (reference range: } \\
0.00 \text { to } 0.49 \text { ) }\end{array}$} & A value of $>1.1 \mathrm{nmol} / \mathrm{L}$ is confirmatory ${ }^{9}$ \\
\hline & & A value of $<0.5$ excludes pheochromocytoma ${ }^{9}$ \\
\hline
\end{tabular}

not report any heritable disorders, nor does he appear to have any CNS disturbance or unusual cutaneous lesions, which makes it unlikely that this disorder is related to his psychiatric symptoms.

A serum metanephrines test measures the metabolites of epinephrine and norepinephrine. These catecholamines are produced in excess by an adrenal or extraadrenal tumor seen in pheochromocytoma. The classic triad of symptoms of pheochromocytoma are hypertension, sweating, and headache; approximately $30 \%$ of patients report significant anxiety and panic (Table 1, ,-7 $^{5}$ page 40). This type of tumor is rare, with an annual incidence of only 2 to 8 cases per 1 million individuals. Among people with hypertension, the annual incidence is $0.1 \%$ to $1.0 \%$, and for those with an adrenal mass, the annual incidence is $5 \%$ (Table 2, ${ }^{6,8}$ page 40 ). Autopsy studies suggest that up to $50 \%$ of pheochromocytomas are undiagnosed. ${ }^{8}$ Left untreated, pheochromocytoma can result in hypertensive crisis, arrhythmia, myocardial infarction, multisystem organ failure, and premature death. ${ }^{7}$ Table $3^{6,7}$ (page 40) highlights some causes of false-positive serum on metanephrines testing.

\section{EVALUATION Metanephrines testing}

Mr. P has what appears to be treatmentresistant hypertension, accompanied by the classic symptoms observed in most patients with pheochromocytoma. Because Mr. $\mathrm{P}$ is participating in the PHP 6 days per week for
6 hours each day, visiting his PCP would be inconvenient, so the treatment team orders the serum metanephrines test. If a positive result is found, Mr. $P$ will be referred to his PCP for further assessment and follow-up care with endocrinology.

\section{TREATMENT Pharmacotherapy to target anxiety and panic}

Next, the treatment team establishes a safety plan for Mr. P, and restarts paroxetine, $20 \mathrm{mg} / \mathrm{d}$, to target his depressed and anxious mood. Alprazolam, $0.5 \mathrm{mg}$ twice daily, is started to target anxious mood and panic symptoms, and to allow time for the anxiolytic properties of the paroxetine to become fully effective. The alprazolam will be tapered and stopped after 2 weeks. Mr. P is started on hydroxyzine, 1 to 2 25-mg tablets 2 to 3 times daily as needed for anxious mood and panic symptoms.

The serum metanephrines test results are equivocal, with a slight elevation of both epinephrine and norepinephrine that is too low to confirm a diagnosis of pheochromocytoma but too elevated to exclude it (Table $4^{9}$ ). With Mr. P's consent, the treatment team contacts his PCP and convey the results of this test. Mr. P schedules an appointment with his PCP for the following week for further assessment and confirmatory pheochromocytoma testing.

After 1 week, Mr. P remains anxious, with a slight reduction in panic attacks from multiple attacks each day to 3 or 4 attacks per week. The team considers adding an additional anxiolytic agent.

\section{Clinical Point}

Mr. P had the classic triad of symptoms of pheochromocytoma: hypertension, sweating, and headache 


\section{Clinical Point}

Several medications, including some psychotropics, can lead to falsepositive results on metanephrines testing

\section{Related Resources}

- National Organization for Rare Disorders. Rare Disease Database: pheochromocytoma. www.rarediseases.org/ rare-diseases/pheochromocytoma/.

- Young WF Jr. Clinical presentation and diagnosis of pheochromocytoma. UpToDate. www.uptodate.com/ contents/clinical-presentation-and-diagnosis-ofpheochromocytoma. Published January 2020.

Drug Brand Names

Alprazolam • Xanax Amitriptyline • Elavil Buspirone • Buspar Carvedilol • Coreg Clonidine - Catapres Doxazosin · Cardura Hydroxyzine • Vistaril

Lisinopril $\cdot$ Prinivil, Zestril
Paroxetine $\cdot$ Paxil
Prazosin $\cdot$ Minipress
Propranolol $\cdot$ Inderal
Terazosin $\cdot$ Hytrin
Trazodone $\cdot$ Desyrel
Zolpidem $\cdot$ Ambien

\section{Which of the following anxiolytic agents} would be the safest option to prescribe until confirmatory testing can confirm or rule out pheochromocytoma?
a) buspirone
b) propranolol
c) trazodone
d) amitriptyline

\section{The author's observations}

The triad of symptoms in pheochromocytoma results directly from the intermittent release of catecholamines into systemic circulation. Surges of epinephrine and norepinephrine lead to headaches, palpitations, diaphoresis, and (less commonly) gastrointestinal symptoms such as nausea, vomiting, and constipation. Persistent or episodic hypertension may be present, with $13 \%$ of patients maintaining a normal blood pressure.-7 Patients with pheochromocytoma-related anxiety typically have substantial or complete resolution of anxiety and panic attacks after tumor resection. $6,8,10$

Because of their ability to raise catecholamine levels, several medications, including some psychotropics, can lead to false-positive results on serum and urine metanephrines testing. Tricyclic antidepressants and beta-blockers can cause false-positive results on plasma assays, while buspirone can cause false-positives on urinalysis assays. ${ }^{5}$ Trazodone, on the other hand, exhibits no catecholaminergic activity and its alpha- 1 adrenergic antagonism may actually have some benefit in pheochromocytoma. ${ }^{11}$ Alpha-1 adrenergic antagonism with doxazosin, prazosin, or terazosin is the first-line of treatment in reducing pheochromocytomarelated hypertension. ${ }^{7}$ Treatment with a betablocker is safe only after alpha-adrenergic blockade occurs. While beta-blockers are useful for reducing the palpitations and anxiety observed in patients with pheochromocytoma, they must not be used alone due to the risk of hypertensive crisis resulting from unopposed alpha-adrenergic agonist activated vasoconstriction. ${ }^{5,7}$

\section{TREATMENT CBT provides benefit}

Mr. $P$ decides against receiving an additional agent for anxiety and instead decides to wait for the outcome of the confirmatory pheochromocytoma testing. He continues to take alprazolam, and both his depressed mood and anxiety improve. His panic attacks continue

\section{Bottom Line}

Pheochromocytoma is a tumor of the adrenal gland. The classic triad of symptoms of this rare condition is hypertension, sweating, and headache; approximately $30 \%$ of patients report significant anxiety and panic. Several medications, including tricyclic antidepressants, beta-blockers, and buspirone, can lead to false-positive results on the serum and urine metanephrines testing used to diagnose pheochromocytoma. 
to lessen, and he appears to benefit from cognitive-behavioral therapy provided during group therapy. Mr. $\mathrm{P}$ is advised by his PCP to taper and stop the alprazolam 3 to 5 days before his 24-hour urine metanephrines test because benzodiazepines can lead to falsepositive results on a urinalysis assay. ${ }^{7}$

\section{OUTCOME Remission of anxiety and depression}

Mr. $P$ has a repeat serum metanephrines test and a 24-hour urinalysis assay. Both are negative for pheochromocytoma. His PCP refers him to cardiology for management of treatment-resistant hypertension. He is discharged from the PHP and continues psychotherapy for depression and anxiety in an intensive outpatient program (IOP). Throughout his PHP and IOP treatments, he continues to take paroxetine and hydroxyzine. He achieves a successful remission of his anxiety and depression, with partial but significant remission of his panic attacks.

\section{The author's observations}

Although Mr. P did not have pheochromocytoma, it is important to rule out this rare condition in patients who present with treatment-resistant hypertension and/or treatment-resistant anxiety.

\section{References}

1. Morrison J. When psychological problems mask medical disorders: a guide for psychotherapists. 2nd ed. New York, NY: The Guilford Press; 2015

2. American Porphyria Foundation. About porphyria. https:// porphyriafoundation.org/patients/about-porphyria. Accessed May 13, 2020

3. Millward L, Kelly P, King A, et al. Anxiety and depression in the acute porphyrias. J Inherit Metab Dis. 2005;28(6): 1099-1107.

4. Bonkovsky H, Maddukuri VC, Yazici C, et al. Acute porphyrias in the USA: features of 108 subjects from porphyria consortium. Am J Med. 2014;127(12): 1233-1241.

5. Tsirlin A, Oo Y, Sharma R, et al. Pheochromocytoma: a review. Maturitas. 2014;77(3):229-238.

6. Leung A, Zun L, Nordstrom K, et al. Psychiatric emergencies for physicians: clinical management and approach to distinguishing pheochromocytoma from psychiatric and thyrotoxic diseases in the emergency room. J Emerg Med. 2017;53(5):712-716

7. Garg M, Kharb S, Brar KS, et al. Medical management of pheochromocytoma: role of the endocrinologist. Indian J Endocrinol and Metab. 2011;15(suppl 4):S329-S336. doi: 10.4103/2230-8210.86976

8. Zardawi I. Phaeochromocytoma masquerading as anxiety and depression. Am J Case Rep. 2013;14:161-163.

9. ARUP Laboratories. Test directory. https://www. aruplab.com. Accessed February 11, 2020.

10. Sriram P, Raghavan V. Pheochromocytoma presenting as anxiety disorder: a case report. Asian J Psychiatr. 2017;29: 83-84.

11. Stahl SM. Stahl's essential psychopharmacology: neuroscientific basis and practical applications. Cambridge, UK: Cambridge University Press; 2013.

\section{Clinical Point}

Rule out pheochromocytoma in patients with treatment-resistant hypertension and/or treatment-resistant anxiety 